\title{
The Heterogenicity of Parotid Gland Squamous Cell Carcinoma: A Study of 49 Patients
}

\author{
ACHIM FRANZEN ${ }^{1}$, ANJA LIEDER $^{1}$, THOMAS GUENZEL ${ }^{2}$ and ANDRE BUCHALI ${ }^{3}$ \\ ${ }^{1}$ Department of Otorhinolaryngology, Head and Neck Surgery, \\ Brandenburg Medical University-Theodor Fontane, Campus Ruppiner Kliniken, Neuruppin, Germany; \\ ${ }^{2}$ ENT Practice Leer, Leer, Germany; \\ ${ }^{3}$ Department of Radio-Oncology and Radiotherapy, \\ Brandenburg Medical University-Theodor Fontane, Campus Ruppiner Kliniken, Neuruppin, Germany
}

\begin{abstract}
Background/Aim: The aim of this study was to critically evaluate diagnostic workup, treatment and outcome of parotid gland squamous cell carcinoma (SCC). Patients and Methods: We retrospectively examined 844 consecutive cases of parotid surgery in a German teaching hospital between 1975 and 2017. Results: A total of 748 patients had parotid surgery. A total of 118 patients (15.7\%) had a malignant tumour. Of those 49 patients had SCC accounting for $6.6 \%$ of all cases, and $41.5 \%$ of all malignant tumours. SCC typically affected males (79.6\%) of advanced age. A total of $86 \%$ of patients had metastatic disease; three cases were primary SCC. Most primary carcinomas were skin $(n=26)$ or pharynx $(n=10)$. Four cases were carcinomas of unknown primary. We performed radical parotidectomy in 47 cases, neck dissections in 42 cases and adjuvant chemoradiotherapy in 44 cases. Conclusion: The majority of parotid SCC are metastases. In cases without known primary lesion and lack of infiltrative tumour growth, a carcinoma with unknown primary should be considered.
\end{abstract}

Malignant tumours of the parotid gland form a heterogeneous group of tumours comprising at least 24 histological types (1). Due to parotid anatomy and lymphatic drainage patterns, metastatic spread from primary tumours outside the gland should be considered, especially in tumours that do not

This article is freely accessible online.

Correspondence to: Achim Franzen, MD, Department of Otorhinolaryngology, Head and Neck Surgery, Brandenburg Medical University-Theodor Fontane, Campus Ruppiner Kliniken, Fehrbelliner Straße 38, 16816 Neuruppin, Germany. Tel: +49 33913947701, Fax: +49 3391393609, e-mail: a.franzen@ruppinerkliniken.de

Key Words: Parotid neoplasm, squamous carcinoma, metastases, unknown primary neoplasms, skin cancer. represent glandular origins, such as malignant melanoma, small cell carcinoma and clear cell carcinoma $(2,3)$.

Squamous cell carcinoma (SCC) of the parotid usually indicates metastatic disease, and diagnosis of primary SCC of the parotid gland requires exclusion of a metastatic process from an extra-glandular primary (4). Parotid metastases commonly originate from tumours of the head and neck, but infra-clavicular primary sites must also be considered. The highest proportion of parotid metastases arise from SCC of the skin of the scalp, face and neck, but their frequency varies among populations in different geographical locations with different levels of sun exposure (5-7). In Europe and the United States, the proportion of metastatic SCC is reported to be around $25 \%$, whilst data from Australia report the proportion of metastatic SCC arising from skin malignancies to be higher than 50\% (57). In metastatic disease, the primary tumour may be diagnosed simultaneously to the metastasis, but in most cases, patients undergo parotid surgery before their primary tumour is diagnosed (8).

Most reports are based on data from clinical centres or pathology institutes (8-10). Population based publications using the Surveillance, Epidemiology and End Results (SEER) database comprise data from more than 2.000 patients each $(11,12)$. Considering the limitations and confines within which published data can be interpreted, primary parotid SCC represents $0.3 \%$ to $6.9 \%$ of all parotid malignancies (12).

Reviewing our results of 844 cases of parotid gland surgery, we investigated disease patterns of primary and metastatic SCC of the parotid gland. The process of diagnostic workup and treatment with focus both on clinical and pathological aspects are critically evaluated.

\section{Patients and Methods}

We retrospectively examined 844 consecutive cases of parotid surgery between 1975 and 2017 at our institution, a large district general and 
academic teaching hospital with a stand-alone Otolaryngology unit in Eastern Germany.

Depending on clinical and histological characteristics, one of the following parotid surgery procedures was performed: superficial, subtotal or total conservative parotidectomy with facial nerve preservation or radical parotidectomy. Open parotid biopsies were performed in a small number of selected cases. Neck dissection was performed contemporaneously in cases with histologically proven SCC. Most patients with malignant tumours received adjuvant radiotherapy with or without concurrent chemotherapy. Histopathological examination was performed in-house, with samples sent to reference laboratories where appropriate. All patient data were obtained by hand searching of patient records and analysed using a statistical software package (Libre Office Calc with R Statistics Package).

Ethical considerations. All investigations and treatments were established clinical practices and were carried out according to accepted clinical practice and in compliance with medical principles of the Declaration of Helsinki and German Federal Law. Informed consent was obtained from all patients prior to treatment. In this retrospective case series, formal ethical approval was not required.

\section{Results}

Overall, 748 patients received surgery for a parotid tumour. Within this cohort, 41 patients had bilateral tumours. A total of 118 tumours out of $748(15.7 \%)$ were malignant and 49 $(6.6 \%)$ were SCC. Therefore, $41.5 \%$ of all malignant parotid tumours were SCC, accounting for the most frequently diagnosed histological entity in this group, followed by malignant lymphoma at $17.8 \%(n=21)$ and carcinoma ex pleomorphic adenoma at $8.5 \%(n=10)$. The proportion of SCC rose from $31.0 \%$ ( 8 out of 26 malignancies) in the $1975-1996$ period to $45.0 \%$ (41 out of 92 malignancies) in the $1996-2016$ period.

SCC affected 39 male patients $(79.6 \%)$ and 10 female patients $(20.4 \%)$ between the ages of 43 and 93 years, with a mean age of 71.8 years. Nine patients were younger than 60 years old and 28 patients were 70 years or older at the time of diagnosis.

A total of 42 patients $(86 \%)$ with SCC had metastatic disease, while only three patients $(6.1 \%)$ were diagnosed with a primary SCC of the parotid gland. Four cases (8.2\%) were classified as carcinomas of an unknown primary (CUP) (Figure 1). In 13 cases of metastatic disease, multiple SCCs were found in one parotid gland and another patient suffered from a metastatic tumour and a Warthin tumour in the same gland.

Primary tumours were located above the clavicle in 40 out of 42 cases $(95.0 \%)$, with 26 of them located in the facial skin or scalp, 10 in the oral cavity or pharynx and 4 in the ear canal. The remaining two primary tumours were located below the clavicle and arose from primary tumours in the lung and the oesophagus (Figure 1).

Patients with primary SCC of the parotid gland received radical parotidectomy with facial nerve sacrifice $(n=2)$ or partial parotidectomy $(n=1)$. Patients with metastatic disease either had a partial parotidectomy $(n=2)$, subtotal parotidectomy $(n=23)$, total parotidectomy $(n=16)$, or a radical parotidectomy with facial nerve sacrifice $(n=3)$. Open biopsy alone was performed in 2 patients.

Excision of skin carcinomas was performed in our patient group in $86 \%$ of cases between 3 and 60 months (average 11.1 months) prior to parotid surgery. These procedures were performed outside our hospital, usually in an out-patient setting, and this information was gathered retrospectively after diagnosis of a parotid metastasis. Patients with metastatic skin cancer were referred to the hospital for excision of a parotid lesion. In such cases, the association between a previously operated skin tumour and the presence of parotid lesions was often not recognized by the referring clinician and therefore not conveyed to the surgeon and often not reported by the patient.

The diagnosis of parotid gland metastasis made simultaneously with the primary tumour was $70 \%$ and $75 \%$ in metastatic disease of SCC of the upper aero-digestive tract and the outer ear canal, respectively.

In the case of oesophageal SCC, parotid gland metastasis was diagnosed 12 months after treating the primary tumour, while in the case of lung cancer the distant metastasis was the first manifestation of disease.

We performed a neck dissection of at least one side of the neck in 42 cases of parotid SCC (86\%). These were all four cases of CUP, four carcinomas of the ear canal, all 10 metastatic pharyngeal cancers and 22 out of 26 metastatic skin cancers $(n=40)$ as well as two out of three primary parotid SCC.

Additional cervical nodal metastases were found in 35 cases ( $83 \%$ of neck dissections): four CUP (100\%), two carcinomas of the ear canal (50\%), 10 upper aero-digestive tract carcinomas $(100 \%), 17$ skin carcinomas $(77.3 \%)$ and two primary SCC (100\%).

Adjuvant radiotherapy of the parotid and neck region or chemo-radiotherapy was performed in 44 out of 49 patients (90\%). The four patients with diagnosis of CUP syndrome received additional radiation of the upper aero-digestive tract at a cumulative dose of 50 Gray.

All patients were observed for at least seven years following parotid surgery. All patients operated in 2012 or later continue to be under clinical observation.

Two out of three patients with primary SCC of the parotid gland $(66.7 \%)$ died within two years after diagnosis from either loco-regional recurrence or disseminated metastatic disease. The other patient was 88 years at the time of diagnosis and died recurrence-free from an illness unrelated to the tumour.

Following the diagnosis of a parotid metastasis, tumour recurrence was observed in 26 out of 46 cases of metastatic tumour $(57.7 \%)$. This included five cases where the tumour 


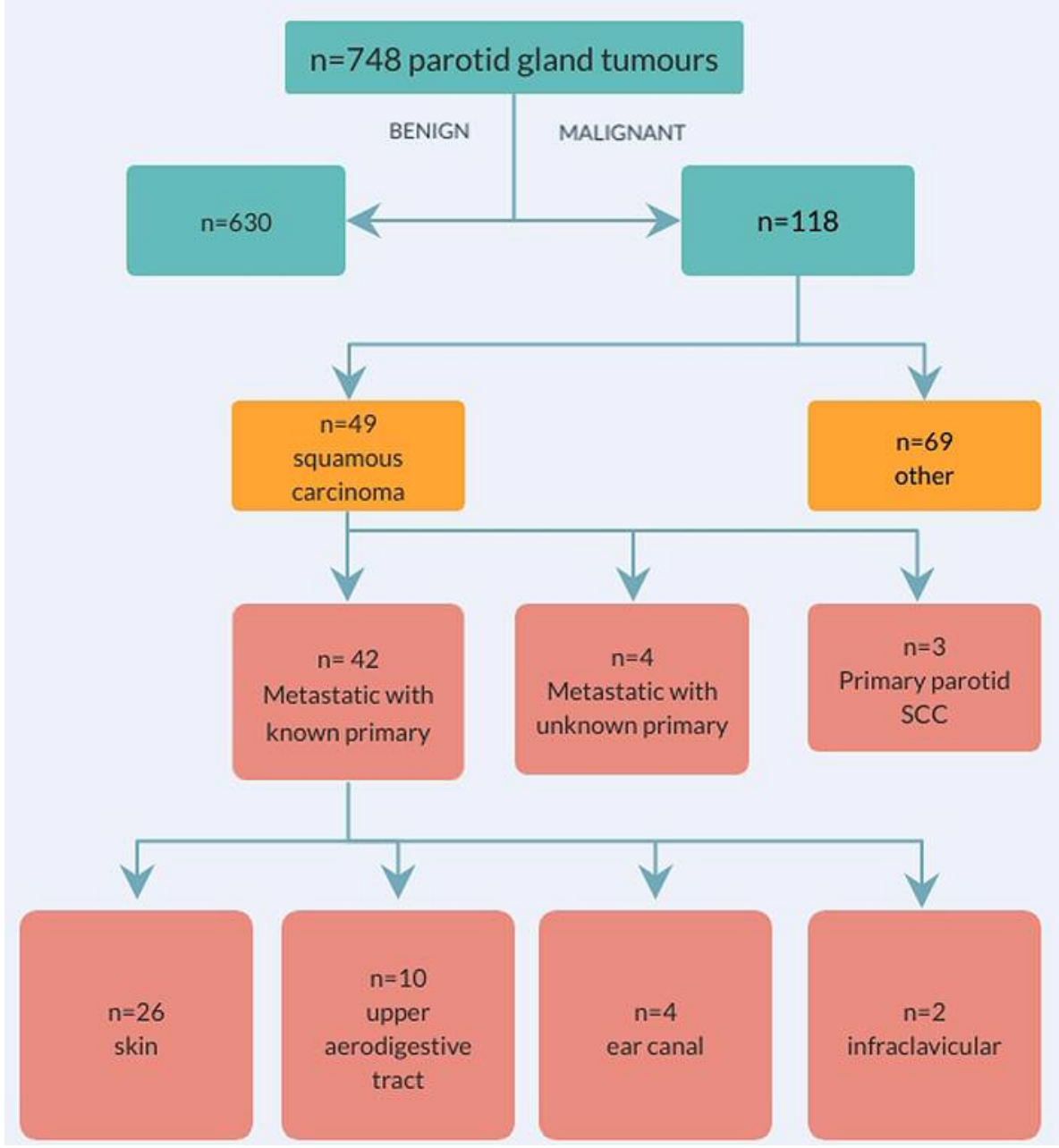

Figure 1. Parotidectomy histopathology by tumour type.

could not be excised completely at the time of surgery and was considered an incomplete resection.

With respect to the site of primary tumour, recurrent disease accounted for 16 out of 26 cases of SCC of the skin (61.5\%), 6 out of 10 cases of upper aero-digestive tract SCC (60.0\%), 2 out of 2 cases of infra-clavicular primary tumour which showed extensive loco-regional and distant spread at the time of diagnosis $(100 \%)$, and one out of four cases with primary SCC of the outer ear canal $(25.0 \%)$. In the four patients with CUP syndrome, local and distant tumour progression was observed in one case $(25 \%)$. The remaining three patients have been free from disease for more than 7 years.

\section{Discussion}

Squamous cell carcinoma (SCC) is an increasingly frequent parotid gland malignancy. Its incidence increased in our patient group over a 40-year period from 31\% between 1975 and 1996 to $45 \%$ between 1997 and 2017. Similar increases were reported in Australia (5), Europe (13), Western China (14) and North America (12). A large population-based study from North America showed that the incidence of SCC of malignant parotid tumours is $20 \%$ of men and $15 \%$ of women (1).

SCCs of the parotid gland are frequently metastases from extra-glandular primary tumours. In our series, $86 \%$ of parotid SCCs were metastatic, while $6 \%$ of parotid SCCs were primary SCCs, the rest were CUP.

Concerning the four cases of unknown primary, we established a separate category: In these patients a primary could not be detected after extensive search, but at the same time their histological characteristics were not typical of a primary SCC (15). Histopathological characteristics of a primary SCC are of a tumour without capsule that originates from the salivary duct epithelium and infiltrates the parotid parenchyma. In just one of our cases, the tumour originated from the intra-glandular Stensen duct.

A close topographical relationship of SCCs to intraglandular lymph nodes without infiltration of the parenchyma 
is a strong indicator for metastatic disease (16). Secondary or metastatic SCC is also likely when direct infiltration from a skin tumour can be demonstrated. Similarly, multiple manifestations of SCC in the parotid gland are highly indicative of metastatic disease. Seifert and colleagues have mentioned that histopathological distinction between primary and secondary SCC may be otherwise problematic (3).

SCC of the parotid gland is aggressive, regardless of whether it is primary or secondary, and requires multimodal therapy including surgery of the parotid gland and neck and adjuvant radiotherapy and/or chemotherapy. The clinical outcome of primary and secondary SCC is similar $(10,12)$. The number of primary SCCs of the present study is too small to compare outcomes.

In the present study, most primary tumours of parotid metastatic disease were located above the clavicle, in the area of lymphatic drainage into parotid lymph nodes. Most common were skin tumours of the face, the outer ear and scalp (62\%). Involvement of the parotid gland was observed following direct infiltration from the buccal skin in two cases. The average period between the diagnosis of the primary tumour and the manifestation of parotid metastasis was 7.5 months. This predominance of metastatic SCC has also been described in multiple studies from Australia where there is high prevalence of cutaneous SCC in a predominantly white population $(5,7,17)$. Similar observations have been made in North America and Europe, where the proportion of parotid metastases is rising $(18,19)$. In many cases, the primary tumour was treated prior to clinical manifestation of parotid metastasis but no connection was made until parotid specimen histopathology $(8,18)$.

SCC of the parotid gland is more common in old age. The peak incidence in our patient group lies in the 7 th and 8 th decade regardless of primary or secondary manifestation (10, 12). Patients with parotid SCC were predominantly male $(79.6 \%)$, in contrast to patients with other malignant parotid tumours, and independently from primary or secondary manifestation. In our study, this may be due to the higher incidence of SCC of skin and upper aero-digestive tract in males. In large mixed-racial population-based studies from North America, white patients account for more than $90 \%$ of parotid SCC $(1,12)$.

In metastatic SCC, location of the primary tumour and presence of metastatic cervical lymph nodes are important prognostic factors. In patients with metastatic cancer to the parotid gland, the overall rate of local recurrence and tumour-associated mortality was $57.7 \%$ of all cases. Taking into account patients with metastases from carcinoma of the skin only, the local recurrence rate increased to $61.5 \%$.

Additional metastatic spread to extra-parotid cervical lymph nodes were an unfavourable prognostic factor. Fifteen out of sixteen patients $(94 \%)$ with skin carcinoma and tumour recurrence had additional cervical metastases.
Elective dissection of the clinically node-negative neck may therefore improve outcome $(10,17)$.

Primary upper aero-digestive tract SCC was the primary tumour in $25 \%$ of patients of the present series. Vice versa, the incidence of parotid metastases in our patients with mucosal SCC of the head and neck was less than $1 \%$ (our clinical audit data - not shown). Parotid metastases from the upper aero-digestive tract are rare compared to those of metastatic skin carcinoma (20). A total of $60 \%$ of our cases with mucosal primary and parotid metastases were found to have widespread disease at time of diagnosis or developed it in the further course of the disease and died from tumour within the observation period, and the general consensus is that prognosis of upper aero-digestive tract metastatic disease tends to be poor (20).

SCC of the ear canal should be differentiated from carcinoma of other locations of the outer ear as it shows different growth patterns and has a poorer overall prognosis (21). In our small group of 4 patients, all primary tumours were treated at an early stage of a T1 primary tumour according to TNM classification, which may explain the more favourable outcome.

Parotid metastases of infra-clavicular primary tumours are often an expression of disseminated metastasis and carry a poor prognosis. Infra-clavicular SCC may be located in the lung, the upper third of the oesophagus, the infra-clavicular skin and extremely rarely in the rectum $(22,23)$.

Primary parotid SCCs have been analyzed in two large epidemiological studies using US population-based databases $(11,12)$ or in retrospective studies from single institutions $(10$, 24). Data for primary parotid SCCs in the US populationbased databases were extracted using the International Classification of Diseases for Oncology in its Third Edition (ICD-O-3), and on the background of the ICD-O-3 alone, differentiation between primary parotid malignancies and parotid metastases is not possible. One of the essential limitations of these studies is that the investigators had no access to original clinical data. Since diagnosis of a primary parotid SCC is a diagnosis that requires exclusion of historical or current SCC elsewhere and pathological examination, it is reasonable to conclude that a significant number of these SCCs may have been metastases $(11,12)$. Another aspect is that the majority of SCC in both these population-based series were white people who generally suffer from cutaneous SCC more frequently. On the other hand, there is no evidence that primary parotid SCC are more frequent in whites than in other racial groups $(1,12)$.

\section{Conclusion}

SCC of the parotid gland is of increasing incidence and is gaining higher significance in the spectrum of malignant parotid neoplasms. The majority of cases are metastatic 
tumours. Often there is excision of a skin SCC in patients medical history, but a connection is often not made as neither clinicians nor patients convey this important medical information to the operating surgeon. Due diligence must be applied and the patient must be carefully questioned about any procedures especially above the clavicle, including cryotherapy and local application of cytostatic agents, however small they may appear. This may help to select the correct staging and surgical procedure and may be an argument for concurrent neck dissection. Frozen section histopathology is also advisable in such cases.

Diagnosis of primary SCC requires clinical exclusion of a primary tumour outside the gland and histological demonstration of infiltrative tumour growth in every single case. Differentiation between primary and secondary SCC on the basis of epidemiological data collections appears not feasible. In cases without a known primary lesion and which lack histological characteristics of infiltrative tumour growth, a metastatic carcinoma with unknown primary should be considered.

\section{Conflicts of Interest}

All Authors declare that they have no conflicting interests regarding this study.

\section{Authors' Contributions}

Achim Franzen conceived and designed the analysis, collected the data and performed the analysis. Anja Lieder contributed analysis tools, contributed to the analysis and write the paper. Thomas Guenzel contributed data. Andre Buchali jointly conceived and designed the analysis with Achim Franzen and oversaw the analysis.

\section{Acknowledgements}

The Authors thank Dr. Julian Seward for his valuable contribution to English language copy editing.

\section{References}

1 Boukheris H, Curtis RE, Land CE and Dores GM: Incidence of carcinoma of the major salivary glands according to the WHO classification, 1992 to 2006: a population-based study in the United States. Cancer Epidemiol Biomarkers Prev 18: 28992906, 2009. PMID: 19861510. DOI: 10.1158/1055-9965. EPI09-0638

2 McKean ME, Lee $\mathrm{K}$ and McGregor IA: The distribution of lymph nodes in and around the parotid gland: an anatomical study. Br J Plast Surg 38: 1-5, 1985. PMID: 3967103.

3 Seifert G, Hennings K and Caselitz J: Metastatic tumors to the parotid and submandibular glands - analysis and differential diagnosis of 108 cases. Pathol Res Pract 181: 684-692, 1986. PMID: 3562340. DOI: 10.1016/S0344-0338(86)80044-9
4 Ellis GL and Auclair P: Malignant lymphoma of the major salivary glands. In: Tumors of the salivary glands. (AFIP Atlas of Tumor Pathology: Series 4). American Registry of Pathology, pp. 453-470, 2008.

5 Bergersen PJ, Kennedy PJ and Kneale KL: Metastatic tumours of the parotid region. Aust N Z J Surg 57: 23-26, 1987. PMID: 3472508. DOI: 10.1111/j.1445-2197.1987.tb01234.x

6 Bron LP, Traynor SJ, McNeil EB and O'Brien CJ: Primary and metastatic cancer of the parotid: comparison of clinical behavior in 232 cases. Laryngoscope 113: 1070-1075, 2003. PMID: 12782825. DOI: 10.1097/00005537-200306000-00029

7 Audet N, Palme CE, Gullane PJ, Gilbert RW, Brown DH, Irish $\mathrm{J}$ and Neligan P: Cutaneous metastatic squamous cell carcinoma to the parotid gland: analysis and outcome. Head Neck 26: 727732, 2004. PMID: 15287040. DOI: 10.1002/hed.20048

8 Ebrahimi A, Clark JR, Ahmadi N, Pame CE, Morgan GJ and Veness MJ: Prognostic significance of disease-free interval in head and neck cutaneous squamous cell carcinoma with nodal metastases. Head Neck 35: 1138-1143, 2013. PMID: 23152126. DOI: 10.1002/hed.23096

9 Brauneis J, Laskawi R, Schröder M and Eilts M: Squamous cell carcinoma in the area of the parotid gland. Metastasis or primary tumor? HNO 38: 292-294, 1990. PMID: 2228743.

10 Ying YL, Johnson JT and Myers EN: Squamous cell carcinoma of the parotid gland. Head Neck 28: 626-632, 2006. PMID: 16475198. DOI: 10.1002/hed 20360

11 Pfisterer MJ, Vazquez A, Mady LJ, Khan MN, Baredes S and Eloy JA: Squamous cell carcinoma of the parotid gland: A populationbased analysis of 2545 cases. Am J Otolaryngol 35: 469-475, 2014. PMID: 24814339. DOI: 10.1016/j.amjoto.2014.03.003

12 Chen MM, Roman SA, Sosa JA and Judson BL: Prognostic factors for squamous cell cancer of the parotid gland: An analysis of 2104 patients. Head Neck 37: 1-7, 2015. PMID: 24339135. DOI: 10.1002/hed.23566

13 Ethunandan M, Pratt CA and Macpherson DW: Changing frequency of parotid gland neoplasms--analysis of 560 tumours treated in a district general hospital. Ann R Coll Surg Engl 84: 16, 2002. PMID: 11890618.

14 Li LJ, Li Y, Wen Y-M, Liu H and Zhao HW: Clinical analysis of salivary gland tumor cases in West China in past 50 years. Oral Oncol 44: 187-192, 2008. PMID: 17418612. DOI: 10.1016/j.oraloncology. 2007.01.016

15 Ellison JM and Auclair P: Primary Squamous Carcinoma. In: Tumors of the Salivary Glands (AFIP Atlas of Tumor Pathology: Series 4). pp. 289-294, 2008.

16 Ellis GL and Auclair P: Malignant Epithelial Neoplasms. In: Tumors of the Salivary Glands (AFIP Atlas of Tumor Pathology: Series 4). pp. 173-386, 2008.

17 O'Brien CJ: The parotid gland as a metastatic basin for cutaneous cancer. Arch Otolaryngol Head Neck Surg 131: 551-555, 2005. PMID: 16027274. DOI: 10.1001/archotol.131.7.551

18 Vauterin TJ, Veness MJ, Morgan GJ, Poulsen MG and O'Brien CJ: Patterns of lymph node spread of cutaneous squamous cell carcinoma of the head and neck. Head Neck 28: 785-791, 2006. PMID: 16783833. DOI: 10.1002/hed.20417

19 O’Hara J, Ferlito A, Takes RP, Rinaldo A, Strojan P, Shaha AR, Rodrigo JP and Paleri V: Cutaneous squamous cell carcinoma of the head and neck metastasizing to the parotid gland - a review of current recommendations. Head Neck 33: 1789-1795, 2011. PMID: 22076982. DOI: $10.1002 /$ hed.21583 
20 Pisani P, Krengli M, Ramponi A, Guglielmetti R and Pia F: Metastases to parotid gland from cancers of the upper airway and digestive tract. Br J Oral Maxillofac Surg 36: 54-57, 1998. PMID: 9578259.

21 Prabhu R, Hinerman RW, Indelicato DJ, Morris CG, Werning JW, Vaysberg $\mathrm{M}$, Amdur RJ, Kirwan $\mathrm{J}$ and Mendenhall WM: Squamous cell carcinoma of the external auditory canal: long-term clinical outcomes using surgery and external-beam radiotherapy. Am J Clin Oncol 32: 401-404, 2009. PMID: 19398900. DOI: 10.1097/COC.0b013e31818f2d48

22 Franzen AM, Günzel T and Lieder A: Parotid gland metastases of distant primary tumours: A diagnostic challenge. Auris Nasus Larynx 43: 187-191, 2016. PMID: 26526643. DOI: 10.1016/j.anl. 2015.09 .010
23 Franzen A, Buchali A and Lieder A: The rising incidence of parotid metastases: Our experience from four decades of parotid gland surgery. Acta Otorhinolaryngol Ital 37: 264-269, 2017. PMID: 28872155. DOI:10.14639/0392-100X-1095

24 Flynn MB, Maguire S, Martinez S and Tesmer T: Primary squamous cell carcinoma of the parotid gland: the importance of correct histological diagnosis. Ann Surg Oncol 6: 768-770, 1999. PMID: 10622505.
Received July 8, 2019

Revised July 22, 2019

Accepted July 23, 2019 